\title{
Enhanced Electromagnetic Interference Shielding in a Duplex-Phase Mg-9Li-3Al-1Zn Alloy Processed by Accumulative Roll Bonding
}

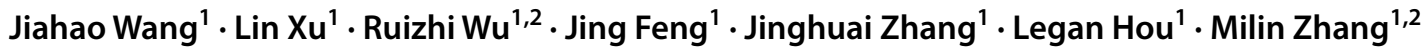

Received: 5 November 2019 / Revised: 7 February 2020 / Published online: 3 March 2020

(C) The Chinese Society for Metals (CSM) and Springer-Verlag GmbH Germany, part of Springer Nature 2020

\begin{abstract}
High electromagnetic shielding performance was achieved in the $\mathrm{Mg}-9 \mathrm{Li}-3 \mathrm{Al}-1 \mathrm{Zn}$ alloy processed by accumulative roll bonding (ARB). The microstructure, electromagnetic interference shielding effectiveness (SE) in the frequency of 30-1500 MHz and mechanical properties of the alloy were investigated. A model based on the shielding of the electromagnetic plane wave was used to theoretically discuss the EMI shielding mechanisms of ARB-processed alloy. Results indicate that the SE of the material increases gradually with the increase in the ARB pass. The enhanced SE can be attributed to the obvious microstructure orientation caused by $\mathrm{ARB}$, and the alternative arrangement of alpha( $\mathrm{Mg})$ phase and beta( $\mathrm{Li})$ phase. In addition, with the increase in ARB pass, the number of interfaces between layers increases and the grain orientation of each layer tends to alignment along $c$-axis, which is beneficial to the reflection loss and multiple reflection loss of the incident electromagnetic wave.
\end{abstract}

Keywords Accumulative roll bonding (ARB) $\cdot \mathrm{Mg}-\mathrm{Li}$ alloy $\cdot$ Dual-phase alloys $\cdot$ Electromagnetic interference $\cdot$ Reflection loss

\section{Introduction}

With the rapid development of electronic and communication technologies, the problem of electromagnetic radiation has become more and more prominent. Electromagnetic radiation not only adversely affects human health and the environment, but also causes interference to electronic equipment and even causes major information losses. Therefore, electromagnetic shielding has received increasing attention, shielding materials play an important role in shielding effects, and the research on shielding materials becomes urgent [1-3].

The electromagnetic shielding effect of materials can be characterized by the shielding effectiveness (SE) [4]. For high-frequency electromagnetic waves, some

Available online at http://link.springer.com/journal/40195.

Ruizhi Wu

rzwu@hrbeu.edu.cn

1 Key Laboratory of Superlight Materials and Surface Technology, Ministry of Education, Harbin Engineering University, Harbin 150001, China

2 College of Science, Heihe University, Heihe 164300, China high-conductivity materials, such as copper, aluminum and magnesium, have a good SE, and for low-frequency electromagnetic waves, high-magnetic permeability materials have good effects, such as ferrite and steel [5-7]. The SE is mainly determined by three mechanisms: the reflection of the electromagnetic wave, called as reflection loss, denoted by R; the absorption of the electromagnetic wave, called as the absorption loss, denoted by $\mathrm{A}$; and the residual energy reflected multiple times in the materials, called as the multiple reflection correction factor, denoted by $\mathrm{B}[8,9]$. In general, electrical shielding is mainly determined by $\mathrm{R}$ and good conductivity promotes electrical shielding; magnetic shielding is mainly determined by A and good magnetic permeability promotes magnetic shielding; and B is mainly determined by the amount of internal interfaces in the material.

Magnesium alloys are always used in shielding enclosures because of the good electromagnetic shielding property and the low density $[10,11]$. As a special magnesium alloy with superlight characteristics, magnesium-lithium alloy has obvious advantages in electromagnetic shielding effectiveness. Based on the $\mathrm{Mg}-\mathrm{Li}$ binary phase diagram [12], Li content between 5.5 and $11 \mathrm{wt} \%$ results in duplex phases, hexagonal close-packed (hcp) structure of $\mathrm{Mg}$ ( $\alpha$-phase) and body-centered cubic (bcc) structure of $\mathrm{Li}$ ( $\beta$-phase). The 
duplex phases in $\mathrm{Mg}-\mathrm{Li}$ alloys bring about a large amount of phase interfaces, which is favor for SE improvement.

ARB process is not only a conventional rolling deformation process, but also a diffusion bonding process. It is widely used in the preparation of ultra-fine grain materials because of its low cost, simple process and being suitable to prepare large-sized ultra-fine grain plate and strip [13]. In addition, ARB process can realize a good physical combination between layers, and the number of layer interfaces sharply increases with the rolling passes. A large number of the physical interfaces forming during ARB process provide many additional reflection interfaces for electromagnetic wave, which is beneficial to the improvement of SE [14].

In this paper, $\mathrm{Mg}-9 \mathrm{Li}-3 \mathrm{Al}-1 \mathrm{Zn}$ alloy, which is composed of duplex matrix phases, was chosen as materials, and the alloy was ARB processed. The aim of the present study is to design and process a kind of $\mathrm{Mg}-\mathrm{Li}$ alloy with both good mechanical properties and electromagnetic shielding. The microstructure, electromagnetic shielding performance and mechanical properties of ARB-processed $\mathrm{Mg}-9 \mathrm{Li}-3 \mathrm{Al}-1 \mathrm{Zn}$ alloy were systematically studied.

\section{Materials and Methods}

Mg-9Li-3 Al-1Zn alloy was prepared from commercial pure magnesium (99.9 wt\%), lithium (99.9 $\mathrm{wt} \%$ ), aluminum (99.9 wt\%) and zinc (99.9 wt\%). The materials were melted in a vacuum induction furnace under the protection of argon atmosphere. The chamber was evacuated to $1 \times 10^{-2} \mathrm{~Pa}$, and then, argon gas was input as a protective gas before melting. The melt temperature was about $700{ }^{\circ} \mathrm{C}$. Then, the melt was poured into a permanent mold. The as-cast ingot was homogenized for $10 \mathrm{~h}$ at $220{ }^{\circ} \mathrm{C}$. Then, the ingot was cut into plates with dimensions of $120 \mathrm{~mm} \times 40 \mathrm{~mm} \times 15 \mathrm{~mm}$. The plates were pre-rolled at $200{ }^{\circ} \mathrm{C}$. The rolling reduction per pass was $10 \%$ until the plates were rolled into sheets with a thickness of $2 \mathrm{~mm}$. In order to investigate the effect of ARB process on the electromagnetic shielding of the $\mathrm{Mg}-9 \mathrm{Li}-3 \mathrm{Al}-1 \mathrm{Zn}$ alloy, three pre-rolled sheets were selected for ARB at $300{ }^{\circ} \mathrm{C}$ with up to three cycles. The schematic of ARB process can be found in our previous literature [15]. Finally, Mg-9Li-3Al-1Zn alloy with a laminated structure of $26\left(3^{3}-1\right)$ interfaces was prepared.

The chemical composition of the studied alloy was tested by an inductively coupled plasma emission spectrometer, which is listed in Table 1. Microstructural observation was performed by optical microscopy (OM) and scanning electron microscopy (SEM). The phase determination and texture measurement were carried out using X-ray diffraction (XRD). As the Mg-9Li-3Al-1Zn alloy consists of hexagonal close-packed (hcp) structure and body-centered cubic (bcc) structure, two sets of pole figures, four incomplete pole
Table 1 Chemical composition of LAZ931 alloy (wt\%)

$\mathrm{Li} \quad \mathrm{Al} \quad \mathrm{Zn} \quad \mathrm{Mg}$

$8.9062 .855 \quad 0.861$ Bal.

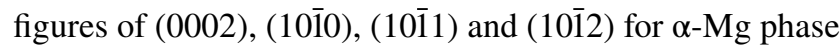
and three incomplete pole figures of (110), (200) and (211) for $\beta$-Li phase were measured.

The mechanical properties were measured with a tensile tester $(1 \mathrm{~mm} / \mathrm{min}$ of the tensile rate). The electrical conductivity of the specimens was measured with a conductivity meter (Sigma 2008B) at ambient temperature. Each conductivity value was the average of five tests. EMI SE was measured using the standard coaxial cable method in accordance with ASTM D4935-2010. The input and output of DN 1015A SE tester are connected to an Agilent E5061B network analyzer. Specimens in the form of disks with $115 \mathrm{~mm}$ in diameter and $2 \mathrm{~mm}$ in thickness were prepared for EMI SE measurement at the frequency of $30-1500 \mathrm{MHz}$.

\section{Results and Discussion}

\subsection{Microstructures}

Figure 1 illustrates the microstructures of the as-rolled $\mathrm{Mg}-9 \mathrm{Li}-3 \mathrm{Al}-1 \mathrm{Zn}$ alloys. It can be observed that $\alpha-\mathrm{Mg}$ phase and $\beta$-Li phase alternatively distribute along the rolling direction. The shape of $\alpha-\mathrm{Mg}$ and $\beta$ - $\mathrm{Li}$ becomes long strip under rolling stress, and many second phases are dispersed in the alloy. It can be seen from the X-ray diffraction pattern that in addition to the diffraction peaks of $\alpha-\mathrm{Mg}$ and $\beta$-Li, there are also diffraction peaks of AlLi phase in the alloy, as shown in Fig. 2.

Three-dimensional optical micrographs of each pass are demonstrated in Fig. 3. With the increase in rolling passes, the laminated structure with an alternate arrangement of the $\alpha-\mathrm{Mg}$ phase and $\beta$-Li phase along RD becomes more obvious, and the spacing between phases in ND decreases gradually. From the microstructure of the RD-TD surface, the $\alpha-\mathrm{Mg}$ phase and the $\beta$-Li phase of the as-rolled alloy are relatively scattered. After ARB1, the $\alpha-\mathrm{Mg}$ phase and $\beta$-Li phase begin to show a distinct directional distribution along RD. With the increase in rolling passes, the directional distribution of $\alpha-\mathrm{Mg}$ phase and $\beta$-Li phase becomes more and more obvious. Meanwhile, from the microstructure of RD-TD and TD-ND, the refinement of the alloy becomes more obvious with the increase in passes. With the increase in ARB passes, the number of interfaces increases. The ARB3 sheet has 26 interfaces, but only two obvious interfaces can be observed in the microstructure, which indicates that the subsequent rolling passes are helpful to 

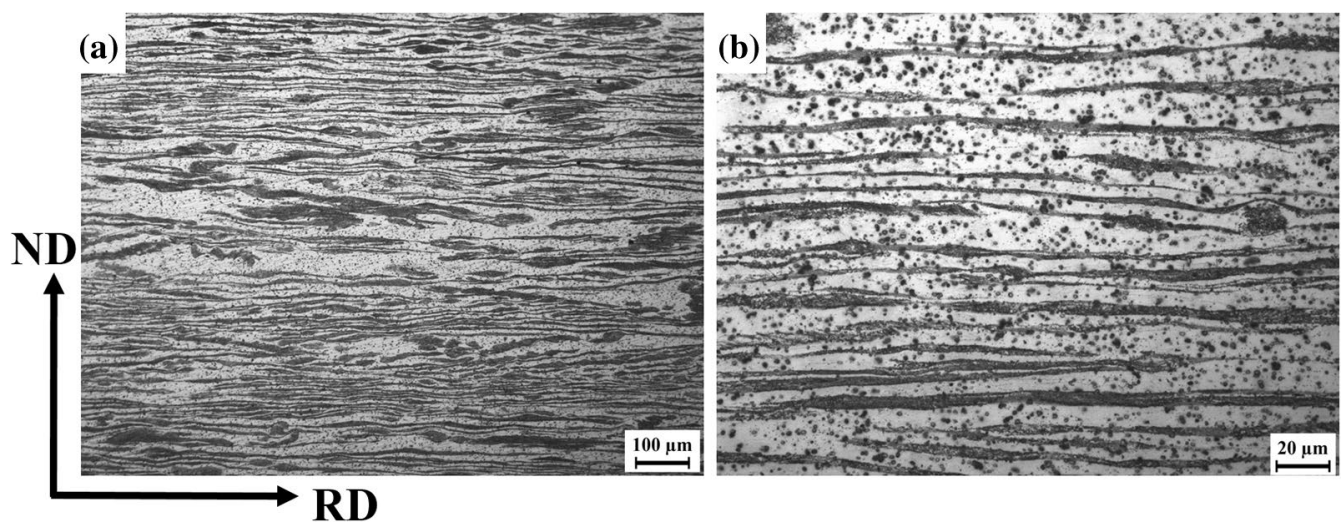

Fig. 1 Microstructures of the as-rolled $\mathrm{Mg}-9 \mathrm{Li}-3 \mathrm{Al}-1 \mathrm{Zn}$ alloy: a low magnification; $\mathbf{b}$ high magnification

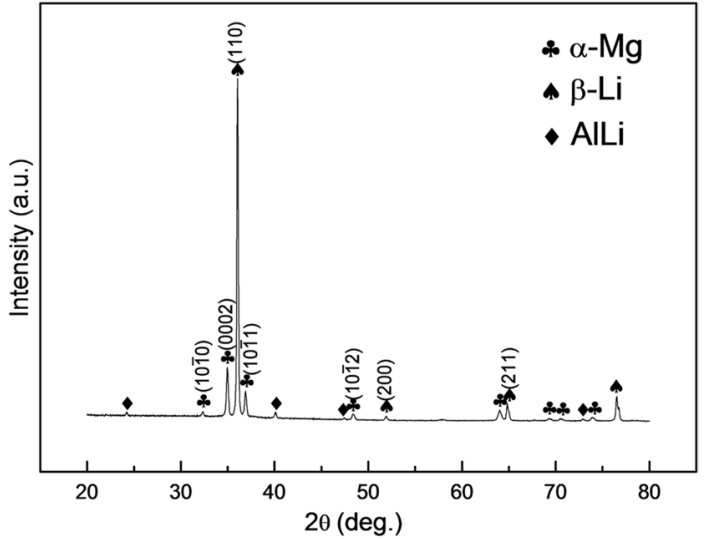

Fig. 2 XRD pattern of the as-rolled $\mathrm{Mg}-9 \mathrm{Li}-3 \mathrm{Al}-1 \mathrm{Zn}$ alloy

further improve the bonding effect of the interfaces formed by previous passes.

Figure 4 shows SEM micrographs of $\mathrm{Mg}-9 \mathrm{Li}-3 \mathrm{Al}-1 \mathrm{Zn}$ alloys in various states. It can be seen that the alloy consists of $\alpha$-Mg phase (light gray), $\beta$-Li phase (dark grey) and some second phases (white). The second phases were confirmed to be AlLi phases by XRD results. The single-layer spacing of the alloy sheets decreases with the progress of ARB, and the single-layer spacing of the ARB3 was about $70 \mu \mathrm{m}$. During the subsequent ARB, under the action of a large pressure, the oxide film forming on the surface of the rough metal was broken and the fresh metal flows out, thereby forming a metallurgical bond at the interfaces. As the rolling cycle progresses, the accumulative deformation gradually increases. Under the action of the stress, the hardened layer, the oxide layer and the pressure bonding region are further broken into finer pressure bonding points and pressure bonding faces. They will begin to flow from the bonding position to the surrounding area in the entire interface plane with the action of the flow stress and finally form a dispersion distribution, as shown in Fig. 4d-f. At the same time, the number of the second phases increases with the increase in the number of passes.

The pole figures of $\alpha-\mathrm{Mg}$ and $\beta-\mathrm{Li}$ in the as-rolled and ARB3-processed $\mathrm{Mg}-9 \mathrm{Li}-3 \mathrm{Al}-1 \mathrm{Zn}$ alloy are shown in Figs. 5 and 6, respectively. In Fig. 5, the texture is a typical rolling texture of magnesium. The maximum polar density is 2.93 , which is distributed in the center of the (0002) base pole figure. In ARB3, the (0002) base pole figure region becomes narrow and dense, and the texture intensity increases to 3.99. This phenomenon indicates that the $c$-axis of the grain gradually becomes parallel to ND. It can be seen from the non-base plane pole figure of the prismatic plane

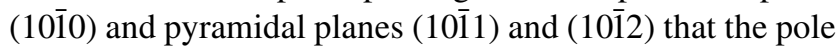
region gradually tends to be more evenly distributed close to the $c$-axis during the deformation process. It is indicated that the prismatic slip and the pyramidal slip are activated during ARB process. Figure 6 shows the (110), (200) and (211) pole figures of the $\beta$-Li phase. The slip of the alloy mainly occurs in the (110) slip plane during the entire deformation process. On the (110) slip plane, the poles are distributed at the center of the circle and at a position of $\pm 30^{\circ}$ in $\mathrm{TD}$, and the texture intensity is higher than that of the other crystal planes. Both the (200) and (211) slip planes are activated. Compared with the as-rolled, the texture intensity of each slip plane of ARB3 decreases, because the initiation of the non-base slip system leads to a decrease in the texture intensity of the base plane to some extent [16-18].

\subsection{Electromagnetic Interference Shielding Effectiveness}

The frequency dependence of EMI shielding effectiveness curves of $\mathrm{Mg}-9 \mathrm{Li}-3 \mathrm{Al}-1 \mathrm{Zn}$ alloy in different passes is illustrated in Fig. 7. The ARB process has a significant effect on the electromagnetic wave shielding. As the number of rolling passes increases, the SE values over the whole test range are significantly improved. Table 2 lists the SE values 
(a)

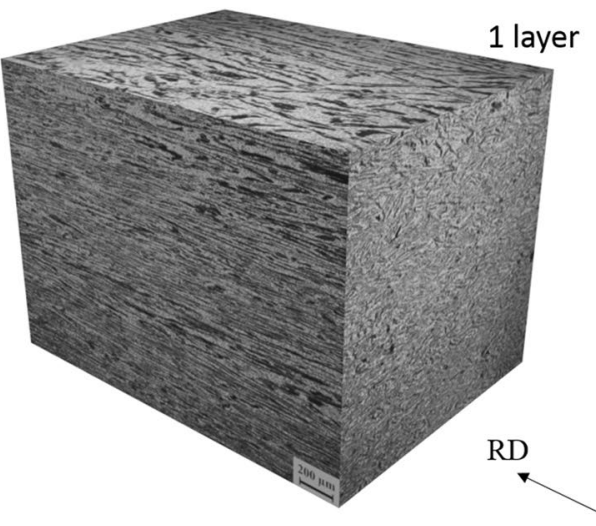

(b)

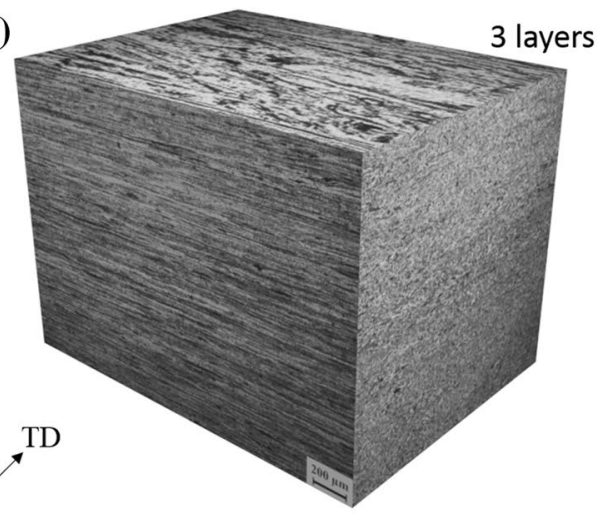

(c)

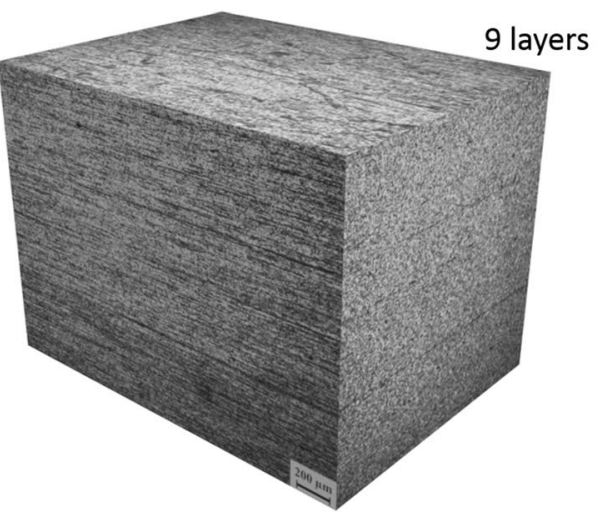

(d)

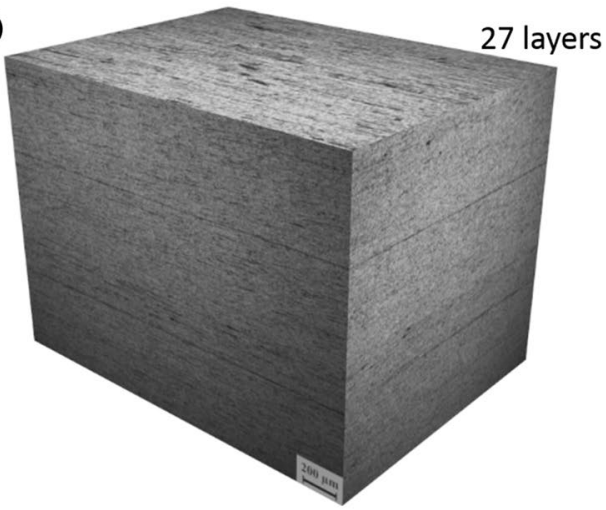

Fig. 3 Three-dimensional optical micrographs of each pass: a as-rolled, b ARB1, c ARB2, d ARB3
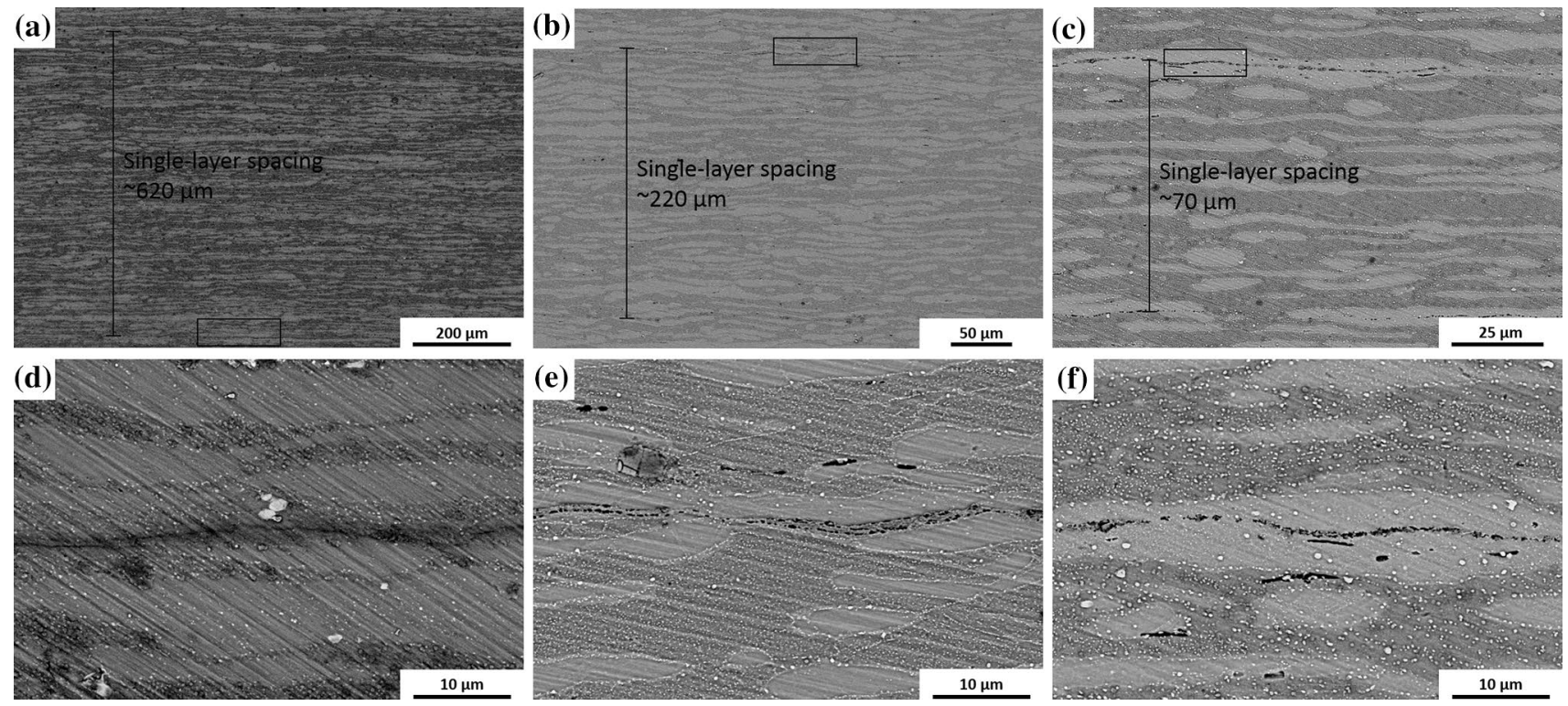

Fig. 4 Typical SEM micrographs showing the microstructures of the alloy in various states: a, d ARB1, b, e ARB2, c, f ARB3

of $\mathrm{Mg}-9 \mathrm{Li}-3 \mathrm{Al}-1 \mathrm{Zn}$ alloy in various states. At $1200 \mathrm{MHz}$, the SE values of the ARB-processed specimens increase by $4.6,8.2$ and $11.1 \mathrm{~dB}$ compared to the as-rolled specimen, respectively. Generally, the industrial requirement of shielding effect is $30-60 \mathrm{~dB}$ and the military requirement is $60-120 \mathrm{~dB}$ [19]. In this work, the sample has excellent shielding at frequencies from 30 to $1500 \mathrm{MHz}$ with SE values between 87 and $107 \mathrm{~dB}$. 

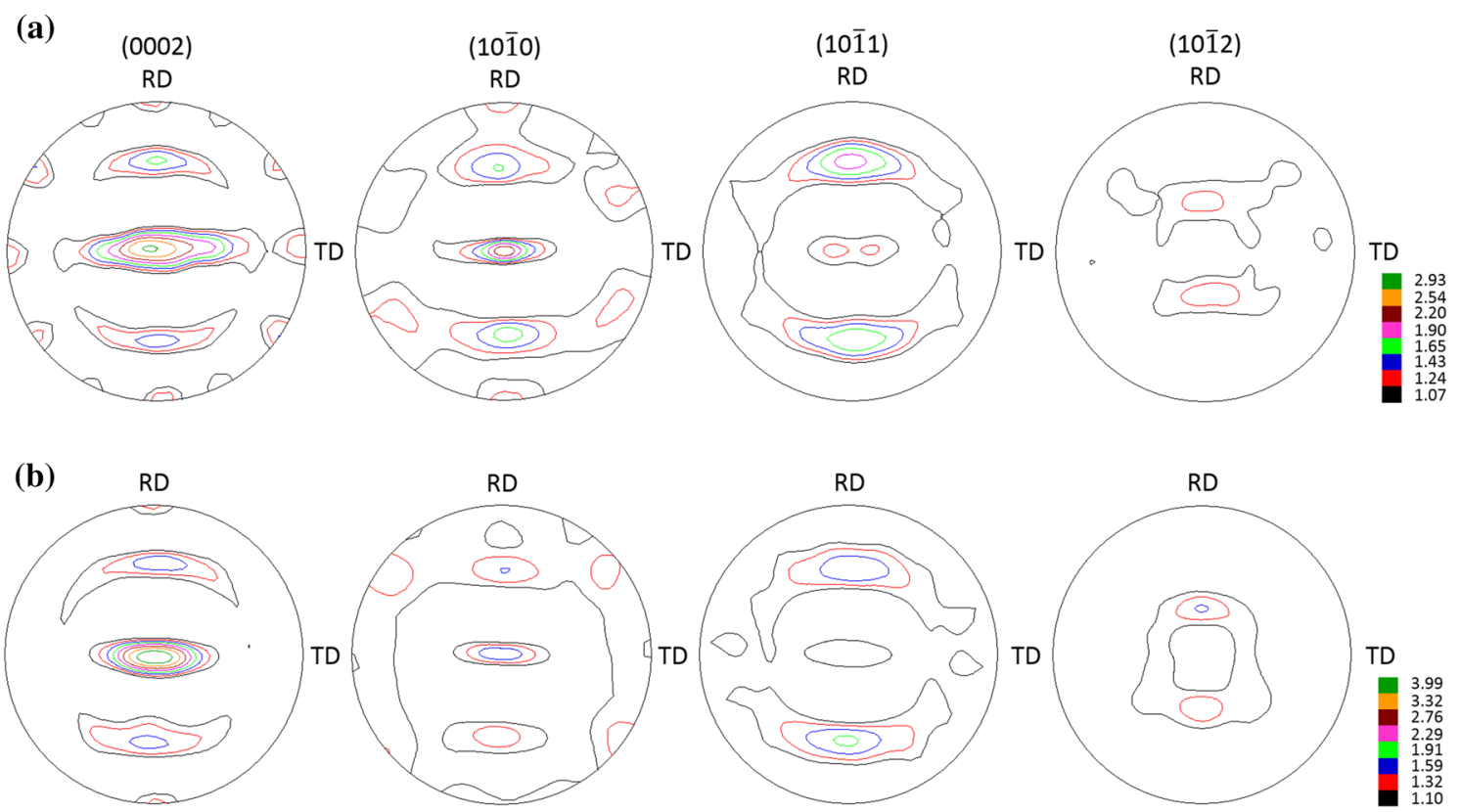

Fig. 5 Pole figures of $\alpha-\mathrm{Mg}$ in $\mathrm{Mg}-9 \mathrm{Li}-3 \mathrm{Al}-1 \mathrm{Zn}$ alloy under different states: a as-rolled; $\mathbf{b}$ ARB3
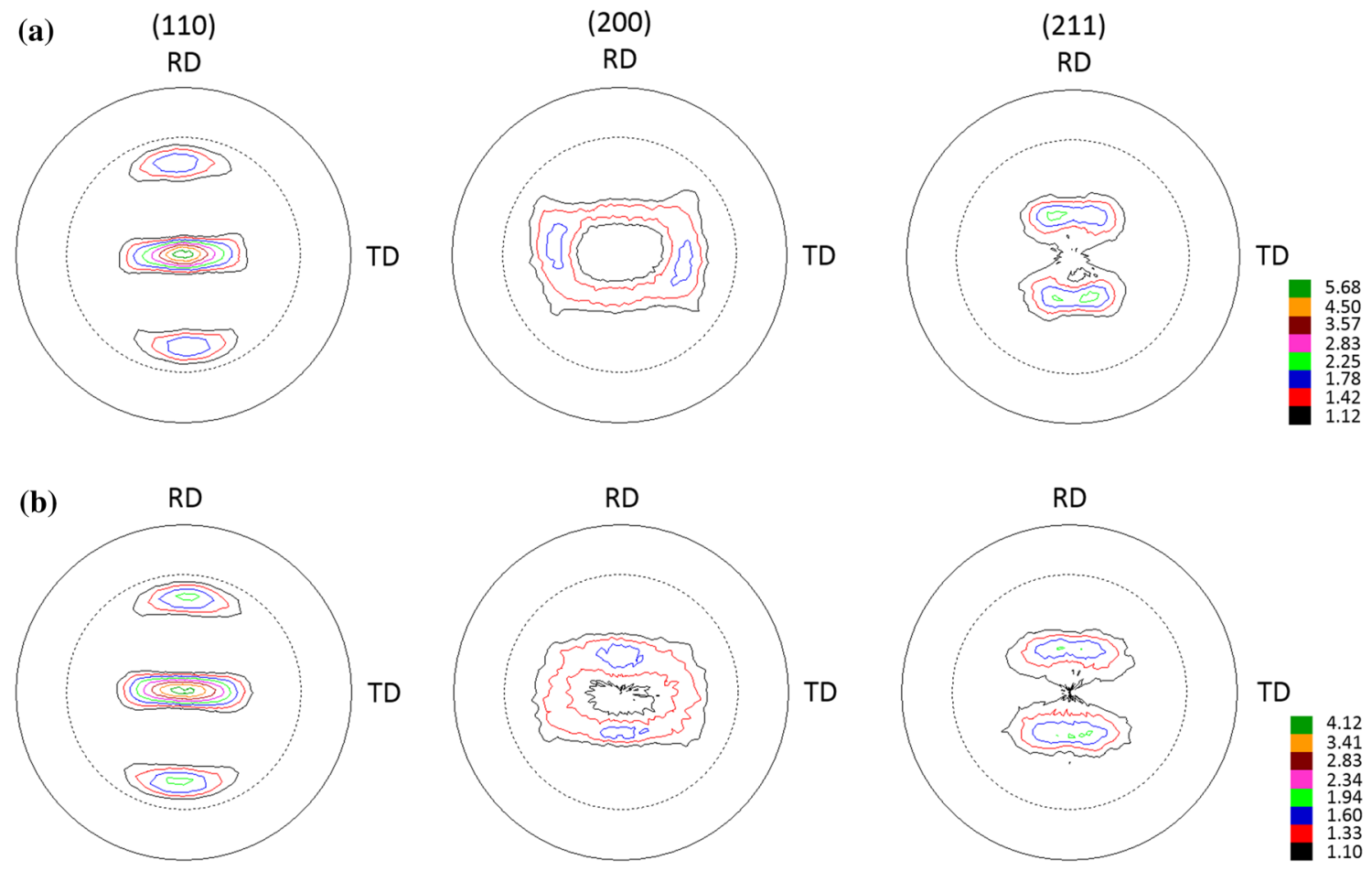

Fig. 6 Pole figures of $\beta$ - $\mathrm{Li}$ in $\mathrm{Mg}-9 \mathrm{Li}-3 \mathrm{Al}-1 \mathrm{Zn}$ alloy under different states: $\mathbf{a}$ as-rolled; $\mathbf{b}$ ARB3 


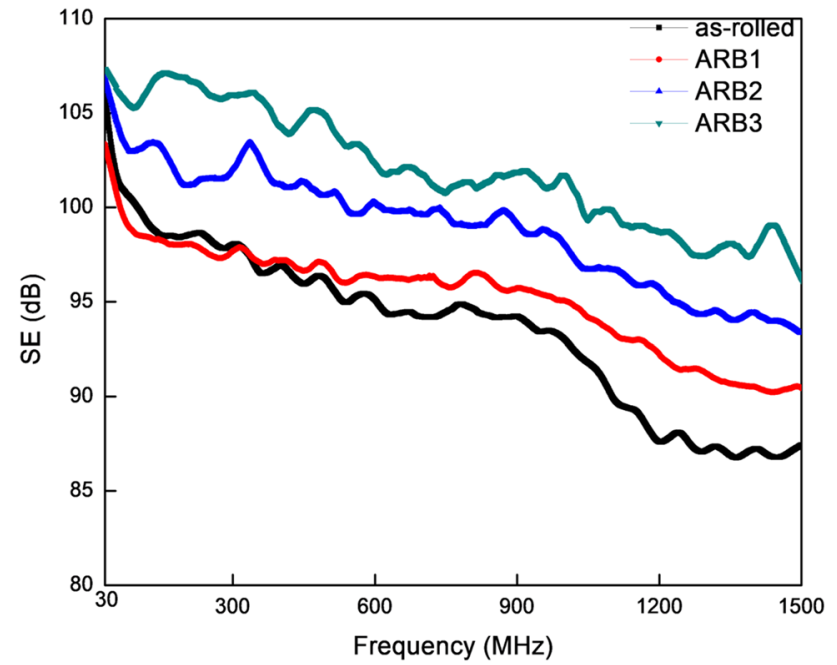

Fig. 7 Electromagnetic shielding effectiveness of accumulative roll bonding alloys during $30-1500 \mathrm{MHz}$ range

Table 2 EMI shielding effectiveness of $\mathrm{Mg}-9 \mathrm{Li}-3 \mathrm{Al}-1 \mathrm{Zn}$ alloy at different frequencies

\begin{tabular}{lccl}
\hline Sample & \multicolumn{3}{l}{ Shielding effectiveness $(\mathrm{dB})$} \\
\cline { 2 - 4 } & $200 \mathrm{MHz}$ & $800 \mathrm{MHz}$ & $1200 \mathrm{MHz}$ \\
\hline As-rolled & 98.5 & 94.7 & 87.6 \\
ARB1 & 98.1 & 96.4 & 92.2 \\
ARB2 & 101.2 & 98.9 & 95.8 \\
ARB3 & 106.9 & 101.4 & 98.7 \\
\hline
\end{tabular}

The multilayer interface electromagnetic shielding mechanisms are shown in Fig. 8a. The whole electromagnetic shielding mechanism is built on a multi-scale basis.

I. The effect of roll bonding interface on electromagnetic shielding performance.

With the increase in ARB process, the number of laminated interfaces increases. The laminated interface can effectively hinder the transmission of electromagnetic waves and play the role of multiple reflection losses, which is beneficial to the increase in shielding effectiveness.

II. The effect of phase interface on electromagnetic shielding performance.

In addition to the multiple reflection loss caused by the phase interface of the second phase [20-22], the $\alpha / \beta$-phase interface characteristic of duplex-phase magnesium-lithium alloy also causes multiple reflection loss. It can be seen from Fig. 3 that the ARB process makes the $\alpha / \beta$-phases in the alloy exhibit an alternate structure, which can play a good role in multiple reflection loss.
III. The effect of grain boundary on electromagnetic shielding performance.

It is well known that the grain size of $\mathrm{Mg}-\mathrm{Li}$ alloy can be refined by ARB [15, 23, 24]. By refining the grain size, a large area of grain boundary can be obtained, so as to increase the multiple reflection loss of the alloy to electromagnetic wave. In addition, grain orientation can also play a good role in the whole electromagnetic shielding process. With the process of ARB, the amount of the grain boundary in the alloy increases, and the orientation of grains tends to be consistent gradually, which makes the incident electromagnetic wave be reflected as much as possible $[3,12]$.

As the transmitted wave from the external surface travels in the whole multilayer shielding material, the amplitude of the wave exponentially decreases due to the coordination of these factors, and the shielding effect is significantly enhanced.

In multilayer electromagnetic wave shielding materials, the shielding mechanism is more complex than that of uniform single shielding material, because it has a huge multi-reflection. At present, the commonly used method for calculating the shielding effectiveness of uniform shielding material is the Schelkunoff formula, which uses a transmission line analogy and is suitable for a conductor flat-type shielding material [25-27]:

$\mathrm{SE}_{\mathrm{dB}}=\mathrm{SE}_{\mathrm{R}}+\mathrm{SE}_{\mathrm{A}}+\mathrm{SE}_{\mathrm{B}}=20 \lg \frac{1}{|T|}$,

$\mathrm{SE}_{\mathrm{R}}=20 \lg \frac{|1+k|^{2}}{4|k|}, \quad k=\frac{Z_{\mathrm{m}}}{\mathrm{Z}_{0}}$,

$\mathrm{SE}_{\mathrm{A}}=20 \lg \left(\mathrm{e}^{\frac{t}{\delta_{\mathrm{m}}}}\right)$,

$\mathrm{SE}_{\mathrm{B}}=20 \lg \left(1-\mathrm{e}^{\frac{-2 t}{\delta_{\mathrm{m}}}}\right)$,

$T=\frac{E_{\mathrm{t}}}{E_{\mathrm{i}}}$ or $\frac{H_{\mathrm{t}}}{H_{\mathrm{i}}}$,

$Z_{\mathrm{m}}=3.68 \times 10^{-7} \sqrt{\frac{f \mu_{\mathrm{m}}}{\sigma_{\mathrm{m}}}}$,

$\delta_{\mathrm{m}}=\sqrt{\frac{1}{\pi \mu_{\mathrm{m}} \sigma_{\mathrm{m}} f}}$, 
(a)

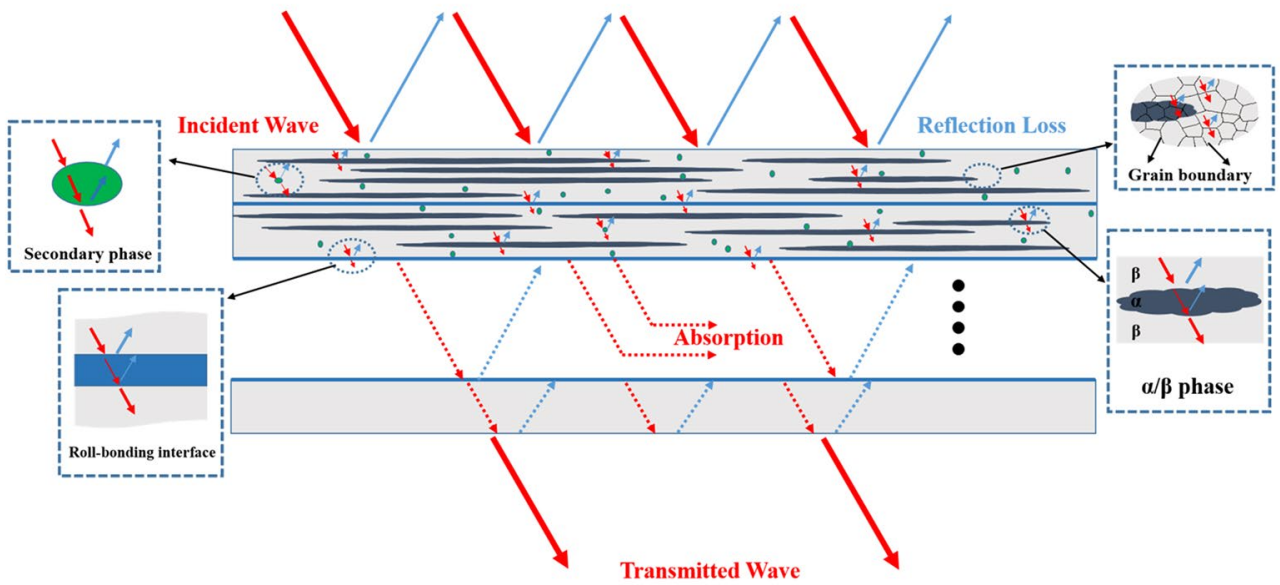

(b)

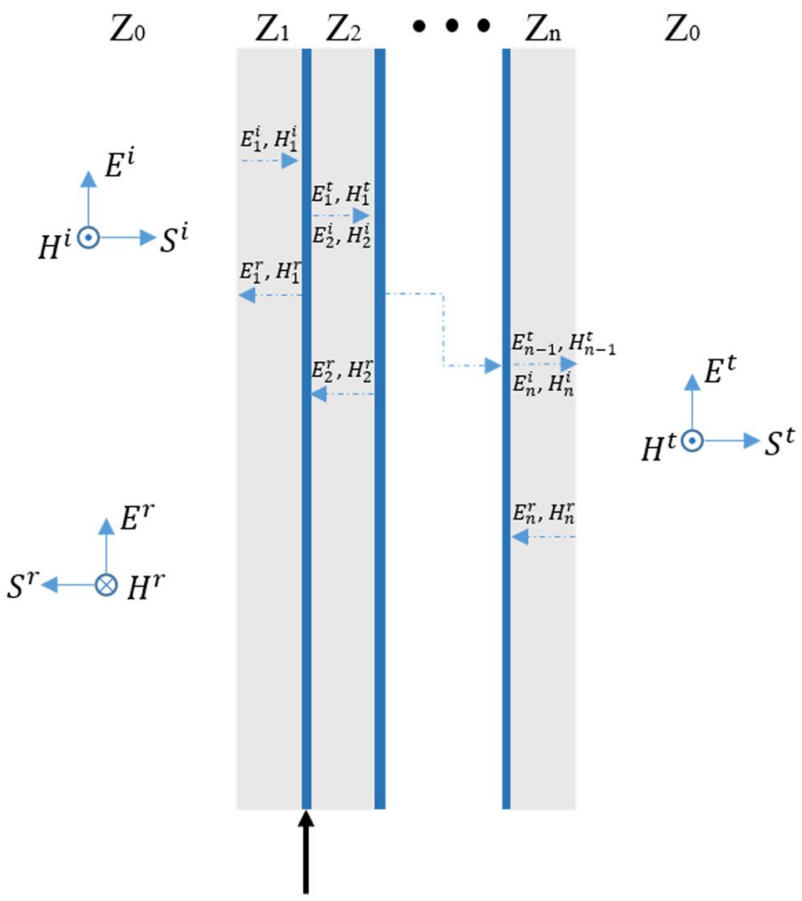

Roll-bonding interface

(oxide layer)

Fig. 8 a Multilayer interface electromagnetic shielding mechanism; b reflection model of electromagnetic waves on a multilayer interface

where $E_{\mathrm{t}}, E_{\mathrm{i}}, H_{\mathrm{t}}, H_{\mathrm{i}}, T, Z_{\mathrm{m}}, \mathrm{Z}_{0}, t, f, \delta_{\mathrm{m}}, \sigma_{\mathrm{m}}$ and $\mu_{\mathrm{m}}$ are the transmission wave electric field intensity, incident wave electric field intensity, transmission wave magnetic field intensity, incident wave magnetic field intensity, transmission coefficient, shield impedance, air impedance (377 $\Omega$ ), thickness, electromagnetic radiation frequency, skin depth, electrical conductivity and magnetic permeability, respectively. $\mathrm{SE}_{\mathrm{R}}$ (reflection loss) is to describe the reflection loss of electromagnetic waves at the two interfaces of the medium, $\mathrm{SE}_{\mathrm{A}}$ (absorption loss) is the absorption loss describing the transmission attenuation of electromagnetic waves in the medium, and $\mathrm{SE}_{\mathrm{B}}$ (multiple reflection loss) is the multiple reflection loss of electromagnetic waves in the medium. On the basis of Eqs. (2) and (6), $\mathrm{SE}_{\mathrm{R}}$ decreases as the electromagnetic radiation frequency increases. For metal shield with good conductivity, its reflection loss $\left(\mathrm{SE}_{\mathrm{R}}\right)$ dominates the whole testing frequency range. The results show that the SE decreases monotonically when the frequency of the incident electromagnetic wave increases. 
Equation (1) shows that the whole shielding effectiveness of the material is related to the transmission coefficient $(T)$. As shown in Fig. 8b, for a multilayer electromagnetic shielding material, it is assumed that the impedance of each layer is $Z_{1}, Z_{2}, Z_{3} \ldots, Z_{n}$ and the air impedance is $Z_{0}$. When electromagnetic waves are incident vertically, the boundary conditions of the interface are continuous, and the electric field and the magnetic field in the medium are continuous on the boundary, that is, $E_{\mathrm{i}}+E_{\mathrm{r}}=E_{\mathrm{t}}$ and $\frac{E_{\mathrm{i}}}{\mathrm{Z}_{0}}+\frac{E_{\mathrm{r}}}{\mathrm{Z}_{0}}=\frac{E_{\mathrm{t}}}{Z_{1}}$, and the formula can be obtained [28-30]:

$R_{1}=\frac{E_{\mathrm{r}}}{E_{\mathrm{i}}}=\frac{Z_{1}-\mathrm{Z}_{0}}{Z_{1}+\mathrm{Z}_{0}}$

$T_{1}=\frac{E_{\mathrm{t}}}{E_{\mathrm{i}}}=\frac{2 Z_{1}}{Z_{1}+\mathrm{Z}_{0}}$

where $R_{1}$ and $T_{1}$ are the reflection coefficients and transmission coefficients of electromagnetic waves at the interface of the material, respectively. According to Eqs. (1) and (9), the reflection loss can be found at:

$$
\begin{aligned}
\mathrm{SE}_{\mathrm{dB}}= & 20 \lg \frac{1}{|T|}=20 \lg \left|\frac{E_{n}}{E_{\mathrm{t}}} \times \frac{E_{n-1}}{E_{n}} \cdots \times \frac{E_{\mathrm{i}}}{E_{1}}\right| \\
& =20 \lg \left|\frac{\mathrm{Z}_{0}+Z_{n}}{2 \mathrm{Z}_{0}} \times \frac{Z_{n}+Z_{n-1}}{2 Z_{n}} \cdots \times \frac{Z_{1}+\mathrm{Z}_{0}}{2 Z_{1}}\right| \\
& =20 \lg \frac{1}{\left|T_{0}\right|}+20 \lg \frac{1}{\left|T_{n}\right|}+20 \lg \frac{1}{\left|T_{1}\right|} \\
& =R_{0}+R_{n}+\cdots+R_{1} .
\end{aligned}
$$

Here, different from the multiple reflection loss B of the single-layer shielding material, the multilayer shielding material can be divided into multiple single-layer shielding materials, and the total reflection loss of the material is equal to the sum of the individual reflection loss of each layer. In the multilayer shielding material prepared by ARB, there will be an oxide layer between the layers, which will cause the impedance imbalance between the layers, and the incident electromagnetic wave will produce reflection loss as a result. The advantage of preparing multilayer material by ARB is that the electromagnetic loss of the material is increased, the electromagnetic wave is absorbed to the maximum extent in the medium, and the internal multilayer structure can play a good role in reflecting the incident electromagnetic wave. The coordination of various factors greatly improves the SE of the materials. The electromagnetic SE obtained in this study is compared with that of the alloys using other techniques in the literature, as shown in Table 3. It can be seen that the electromagnetic shielding performance of $\mathrm{Mg}-9 \mathrm{Li}-3 \mathrm{Al}-1 \mathrm{Zn}$ alloy processed by ARB is very excellent.

\subsection{Mechanical Properties}

Figure 9 shows the tensile properties of the ARB-processed Mg-9Li-3Al-1Zn sheets. The yield strength and tensile strength of the as-rolled alloys are $152 \mathrm{MPa}$ and $194 \mathrm{MPa}$, respectively, and the elongation is $24.1 \%$. Compared with the as-rolled alloy, ARB-processed $\mathrm{Mg}-9 \mathrm{Li}-3 \mathrm{Al}-1 \mathrm{Zn}$ alloy has better mechanical properties. The yield strength and tensile strength of ARB1 alloy are $188 \mathrm{MPa}$ and $215 \mathrm{MPa}$, respectively. With the increase in ARB pass, the strength of the alloy gradually increases. After three passes, the yield strength and tensile strength of the alloy increase to $208 \mathrm{MPa}$ and $241 \mathrm{MPa}$, respectively. At the same time, as the ARB pass increases, the elongation of the alloy gradually decreases, which is caused by the work hardening effect.

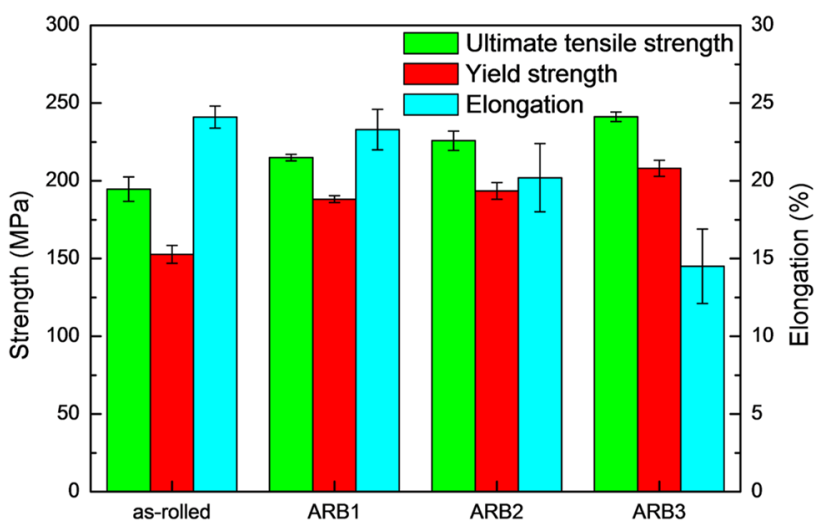

Fig. 9 Tensile properties of the ARB-processed $\mathrm{Mg}-9 \mathrm{Li}-3 \mathrm{Al}-1 \mathrm{Zn}$ sheets
Table 3 Comparison of the electromagnetic shielding effectiveness for several alloys prepared by different processes

\begin{tabular}{lllll}
\hline Alloys & Processing method & $\begin{array}{l}\text { SE }(\mathrm{dB}) \\
f=200 \mathrm{MHz}\end{array}$ & $\begin{array}{l}\text { SE (dB) } \\
f=1200 \mathrm{MHz}\end{array}$ & References \\
\hline Mg-9Li-3Al-1Zn & ARB & 106.9 & 98.7 & This work \\
AZ31 magnesium alloy & As-rolled & $\sim 95$ & $\sim 90$ & {$[3]$} \\
Al2024/cenospheres composite & Squeeze casting & 37 & 35 & {$[31]$} \\
1J50 magnetic alloy & Squeeze casting & 80 & 68 & {$[31]$} \\
ZK60 magnesium alloy & As-extruded & 60 & 56 & {$[21]$} \\
Pure magnesium & As-cast & $\sim 61$ & $\sim 53$ & {$[32]$} \\
\hline
\end{tabular}


After three passes, the elongation of the alloy decreases to $14.5 \%$.

In the process of $\mathrm{ARB}$, the main strengthening mode of the alloy can be explained as strain hardening, dispersion strengthening and refinement strengthening. According to previous studies $[15,33]$, refinement strengthening always acts on the whole plastic deformation process. The principle of strain hardening is based on the dislocation multiplication, dislocation motion and mutual dislocation interaction [34-37]. During ARB process, the formation of a large number of dislocations restricts the base slip and increases the intensity of basal texture (Fig. 5). Meanwhile, a large dislocation pileup will be produced near the bonding layer, which will generate a shear stress field near the roll bonding interface, and this stress field is expected higher than that from ordinary pileups at conventional grain boundaries [38]. With the progress of the ARB process, the interfaces between adjacent layers interact with each other, promoting strain strengthening. In addition to strain hardening, dispersed precipitates also contribute to the strengthening. AlLi phases are crashed into smaller particles and distribute evenly in the matrix during ARB process (Fig. 4). The uniformly distributed particles can serve as crack source and barrier to hinder the movement of dislocations during tensile testing [39]. Under the joint action of the strengthening mechanisms, the strength of the alloy continues to increase, and the decreasing trend of the elongation gradually decreases.

\section{Conclusions}

1. The as-rolled $\mathrm{Mg}-9 \mathrm{Li}-3 \mathrm{Al}-1 \mathrm{Zn}$ alloy is composed of $\alpha-\mathrm{Mg}, \beta-\mathrm{Li}$ and AlLi phases. AlLi phases are uniformly distributed in the $\beta$-phase. The interface bonding introduced in the previous pass can be fully improved by the subsequent ARB passes.

2. With the increase in ARB passes, the shielding efficiency of $\mathrm{Mg}-9 \mathrm{Li}-3 \mathrm{Al}-1 \mathrm{Zn}$ alloy increases significantly in the whole testing frequency of 30-1500 MHz. The ARB3 sample displayed better EM shielding (96-107 dB). The main shielding mechanism of $\mathrm{Mg}-9 \mathrm{Li}-3 \mathrm{Al}-1 \mathrm{Zn}$ alloy processed by ARB is the reflection loss provided by the roll bonding interface and the multiple reflection loss provided by the dual-phase matrix and grain boundary.

3. With the progress of ARB, the strength of the alloy is significantly improved, and the UTS of ARB3 is the highest, reaching about $241 \mathrm{MPa}$, while the elongation decreases from 24.1 to $14.5 \%$ due to the strain hardening and dispersed AlLi phase.

Acknowledgements This work was financially supported by the National Natural Science Foundation of China (Nos. 51671063,
51771060, 51871068, 51971071), the Domain Foundation of Equipment Advance Research of 13th Five-year Plan (No. 61409220118), the Fundamental Research Funds for the Central Universities (No. HEUCFG201834) and the Harbin City Application Technology Research and Development Project (No. 2017RAQXJ032).

\section{References}

[1] J.M. Thomassin, C. Jérôme, T. Pardoen, C. Bailly, I. Huynen, C. Detrembleur, Mater. Sci. Eng., R 74(7), 211 (2013)

[2] M.S. Ozen, E. Sancak, N. Soin, T.H. Shah, A. Zarei, E. Siores, Fibers Polym. 19(2), 321 (2018)

[3] K. Song, F.S. Pan, X.H. Chen, Z.H. Zhang, A.T. Tang, J. She, Z.W. Yu, H.C. Pan, X.Y. Xu, Mater. Lett. 157, 73 (2015)

[4] L.Z. Liu, X.H. Chen, J.F. Wang, L.Y. Qiao, S.Y. Gao, K. Song, C.Y. Zhao, X.F. Liu, D. Zhao, F.S. Pan, J. Mater. Sci. Technol. 35(6), 1070 (2019)

[5] N. Joseph, M.T. Sebastian, Mater. Lett. 90, 64 (2013)

[6] K. Song, F.S. Pan, X.H. Chen, A.T. Tang, H.C. Pan, S. Luo, Mater. Res. Innov. 18(S4), 193 (2014)

[7] B.J. Wang, D.K. Xu, S.D. Wang, L.Y. Sheng, R.C. Zeng, E.H. Han, Int. J. Fatigue 120, 46 (2019)

[8] Z. Luo, X.H. Chen, K. Song, C.Q. Liu, Y. Dai, D. Zhao, F.S. Pan, Acta Metall. Sin. (Engl. Lett.) 32(7), 817 (2019)

[9] J. Wu, D.D.L. Chung, Carbon 40(3), 445 (2002)

[10] B.Y. Qian, W. Miao, F. Min Qiu, D.H. Gao, J.F. Hu, R.Z. Sun, B. Wu, S.Betsofen Krit, Acta Metall. Sin. (Eng. Lett.) 32, 194 (2019)

[11] J.H. Zhang, S.J. Liu, R.Z. Wu, L.G. Hou, M.L. Zhang, J. Magnes. Alloys 6, 277 (2018)

[12] J.H. Wang, R.Z. Wu, J. Feng, J.H. Zhang, L.G. Hou, M.L. Zhang, Mater. Charact. 157, 109924 (2019)

[13] H.J. Wu, T.Z. Wang, R.Z. Wu, L.G. Hou, J.H. Zhang, X.L. Li, M.L. Zhang, J. Manuf. Process. 46, 139 (2019)

[14] J. Hou, Q.L. Zhang, Y.X. Niu, F.K. Ning, Q.C. Le, Mater. Res. Express. 5(6), 066533 (2018)

[15] T.Z. Wang, H.P. Zheng, R.Z. Wu, J.L. Yang, X.D. Ma, M.L. Zhang, Adv. Eng. Mater. 18(2), 304 (2016)

[16] S.Q. Yin, W.C. Duan, W.H. Liu, L. Wu, J.M. Yu, Z.L. Zhao, M. Liu, P. Wang, J.Z. Cui, Z.Q. Zhang, Corros. Sci. 60(8), 108419 (2019)

[17] W.H. Liu, X. Liu, C.P. Tang, W. Yao, Y. Xiao, X.H. Liu, J. Magnes. Alloys 6, 77 (2018)

[18] X.Y. Liu, L.W. Lu, K. Sheng, T. Zhou, Acta Metall. Sin. (Engl. Lett.) 32(6), 710 (2019)

[19] Y. Gao, L. Huang, Z.J. Zheng, H. Li, M. Zhu, Appl. Surf. Sci. 253(24), 9470 (2007)

[20] S.Y. Gao, X.H. Chen, F.S. Pan, K. Song, C.Y. Zhao, L.Z. Liu, X.F. Liu, D. Zhao, Sci. Rep. 8, 1625 (2018)

[21] X.H. Chen, J. Liu, Z.H. Zhang, F.S. Pan, Mater. Des. 42, 327 (2012)

[22] X.H. Chen, J. Liu, F.S. Pan, J. Phys. Chem. Solids 74(6), 872 (2013)

[23] L.G. Hou, T.Z. Wang, R.Z. Wu, J.H. Zhang, M.L. Zhang, A.P. Dong, B.D. Sun, S. Betsofen, B. Krit, J. Mater. Sci. Technol. 34, 317 (2018)

[24] F. Zhong, T.Z. Wang, R.Z. Wu, L.G. Hou, J.H. Zhang, M.L. Zhang, X.L. Li, A.P. Dong, B.D. Sun, Adv. Eng. Mater. 19(5), 1600817 (2017)

[25] M.Y. Li, S. Gupta, C. Chang, N.H. Tai, Compos. Part B 161, 617 (2019)

[26] R.B. Schulz, V.C. Plantz, D.R. Brush, IEEE Trans. Electromagn. Compat. 30(3), 187 (1988)

[27] H.T. Guan, S.H. Liu, Y.P. Duan, J. Cheng, Cem. Concr. Compos. 28(5), 468 (2006) 
[28] Z.M. Song, L. Wang, X.L. Gao, Z.Y. Wang, Inf. Technol. 34(6), $172(2010)$

[29] P. Saini, M. Arora, New Polym. Specif. Appl. 3, 73 (2012)

[30] Y. Naito, K. Suetake, IEEE Trans. Microw. Theory Technol. 19(1), 65 (1971)

[31] G.H. Wu, X.L. Huang, Z.Y. Dou, S. Chen, L.T. Jiang, J. Mater. Sci. 42(8), 2633 (2007)

[32] Z.H. Zhang, F.S. Pan, X.H. Chen, J. Liu, J. Mater. Eng. 3(1), 52 (2013)

[33] H.J. Wu, T.Z. Wang, R.Z. Wu, L.G. Hou, J.H. Zhang, X.L. Li, M.L. Zhang, J. Mater. Process. Technol. 254, 265 (2018)
[34] F. Zhong, H.J. Wu, Y.L. Jiao, R.Z. Wu, J.H. Zhang, L.G. Hou, M.L. Zhang, J. Mater. Sci. Technol. 39, 124 (2020)

[35] Q. Ji, Y.J. Ma, R. Wu, J.H. Zhang, L.G. Hou, M.L. Zhang, J. Alloys Compd. 800, 72 (2019)

[36] M. Duan, L. Luo, Y. Liu, J. Alloys Compd. 823, 153691 (2020)

[37] Y. Wang, Z. Zhang, R.Z. Wu, J.F. Sun, Y.L. Jiao, L.G. Hou, X.L. Li, J.H. Zhang, M.L. Zhang, Mater. Sci. Eng., A 745, 411 (2019)

[38] X.L. Ma, C.X. Huang, J. Moering, M. Ruppert, H.W. Hoppel, M. Goken, J. Narayan, Y.T. Zhu, Acta Mater. 116, 43 (2016)

[39] W.C. Liu, S. Feng, Z. Li, J. Zhao, G.H. Wu, X.F. Wang, L. Xiao, W.J. Ding, J. Mater. Sci. Technol. 34(12), 2256 (2018) 